\title{
Effects of local anaesthetic dilution on the characteristics of ultrasound guided axillary brachial plexus block: a randomised controlled study
}

\author{
Anil Ranganath, Osman Ahmed, Gabriella Iohom
}

Department of Anaesthesia and Intensive Care Medicine, Cork University Hospital, Cork, Ireland

\begin{abstract}
Aims: Ultrasound guidance has led to marked improvement in the success rate and characteristics of peripheral nerve blocks. However, effects of varying the volume or concentration of a fixed local anaesthetic dose on nerve block remains unclear. The purpose of our study was to evaluate whether at a fixed dose of lidocaine, altering the volume and concentration will have any effect on the onset time of ultrasound-guided axillary brachial plexus block. Material and methods: Twenty patients were randomised to receive an ultrasound-guided axillary brachial plexus block with either lidocaine $2 \%$ with epinephrine (20 $\mathrm{ml}$, Group 2\%) or lidocaine 1\% with epinephrine (40 ml, Group 1\%). The primary endpoint was block onset time. Secondary outcomes included duration of the block, performance time, number of needle passes, incidence of paraesthesia and vascular puncture. Results: The median [IQR] onset time of surgical anaesthesia was shorter in Group 1\% when compared to Group $2 \%(6.25$ [5-7.5] min vs 8.75 [7.5-10] min; $\mathrm{p}=0.03)$. The mean (SD) overall duration of surgical anaesthesia was significantly shorter in Group 1\% compared to Group 2\% (150.9 $\pm 17.2 \mathrm{~min}$ vs $165.1 \pm 5.9 \mathrm{~min} ; \mathrm{p}=0.02)$. Group 1\% had a shorter performance time with fewer needle passes. The incidence of vascular puncture and paraesthesia was similar in the two groups. Conclusion: Ultrasound-guided axillary brachial plexus blocks performed using a higher volume of lower concentration lidocaine was associated with shorter onset time and duration of surgical anaesthesia.
\end{abstract}

Keywords: brachial plexus block; axillary; ultrasound; local anaesthetic; lidocaine

\section{Introduction}

Ultrasound-guided axillary brachial plexus block (USgABPB) is an effective and reliable technique for the provision of surgical anaesthesia for forearm and hand surgeries [1-4]. Previously, numerous studies have compared efficacy of brachial plexus block using different local anaesthetic solutions of varying concentrations and volumes [5-7]. However, only few studies have shown that, at constant dose, altering the volume or concen-

Received 02.02.2021 Accepted 05.06.2021

Med Ultrason

2022, Vol. 24, No 1, 38-43

Corresponding author: Dr Gabriella Iohom PhD

Department of Anaesthesia and Intensive Care

Medicine, Cork University Hospital,

Wilton Road, Cork, Ireland

Phone: +353214922135

Fax: +353214643454

E-mail: giohom@ucc.ie tration can affect the characteristics of the nerve block. Historically, it has been reported that higher concentration/lower volume yielded a shorter onset time when compared to higher volume/lower concentration solution using a single injection nerve stimulation technique for sciatic nerve block $[8,9]$. In contrast, in perivascular axillary blocks with a fixed dose of local anaesthetic, larger volumes provided a better quality sensory [10] and quicker onset motor block [11] when compared to lower volumes. The results from these studies were inconsistent with respect to onset time, success rate and duration of the block. In addition, it is unknown whether they can be replicated with ultrasound guidance. We have chosen to focus on the USgABPB with lidocaine plus epinephrine which is offered preferentially to ambulatory upper limb trauma patients at our institution [12].

In this prospective, randomised, double-blind study, we examined whether two different volumes and concentrations of a fixed dose of lidocaine with epinephrine 
influenced the characteristics of USgABPB. We hypothesised that $40 \mathrm{~mL}$ of lidocaine $1 \%$ with epinephrine would result in a shorter onset time when compared to $20 \mathrm{~mL}$ of lidocaine $2 \%$ with epinephrine.

\section{Material and methods}

This single centre study was approved by the Clinical Research Ethics Committee of Cork Teaching Hospitals, Cork, Ireland [ECM 4(mm) 01/07/14; 01 July 2014, Chairperson Professor Michael G Molloy], registered at https://clinicaltrials.gov (NCT03207035), and carried out at Cork University Hospital. Having obtained written informed consent from each, patients aged 18 years or older, ASA grade I-III scheduled to undergo minor unilateral upper limb trauma surgery of the hand or forearm, were enrolled in the study. Exclusion criteria were contraindication to regional anaesthesia, hypersensitivity to amide local anaesthetics, intolerance, or contraindication to non-steroidal anti-inflammatory drugs, BMI $>35$, pregnancy, cardiac conduction abnormalities, history of hepatic and renal impairment, chronic pain, neuromuscular disease, and psychiatric disorder.

Patients were randomised using computer-generated sequence of random numbers and sealed envelope technique, prepared by an investigator with no clinical involvement in the trial. They were subsequently allocated to receive USgABPB with either $20 \mathrm{~mL}$ lidocaine $2 \%$ with 1:200,000 epinephrine (Group 2\%) or $40 \mathrm{~mL}$ lidocaine 1\% with 1:400,000 epinephrine (Group 1\%) (diluted up to the study volume with $0.9 \%$ saline). Intravenous access was established in the contralateral upper limb and standard monitoring was employed throughout the procedure. The operative arm was abducted and externally rotated with the elbow flexed at $90^{\circ}$. Under aseptic precautions the axillary brachial plexus block was performed under ultrasound guidance alone using a SonoSite Titan unit (SonoSite ${ }^{\circledR}$, Bothwell, WA) with a $38 \mathrm{~mm}$ linear array $5-10 \mathrm{MHz}$ transducer (L38). Following the identification of the median, ulnar, radial, and musculocutaneous nerves in the axillary region, a 50 $\mathrm{mm}$ 24-gauge insulated short bevel needle (Stimuplex ${ }^{\circledR}$ B. Braun, Melsungen, Germany) was advanced in-plane towards each nerve with the aim of surrounding it with either $5 \mathrm{~mL}$ (Group 2\%) or $10 \mathrm{~mL}$ (Group 1\%) of local anaesthetic solution. Dynamic manipulation of the needle was sought to facilitate the circumferential perineural spread of local anaesthetic. All blocks were performed by an operator experienced in USgABPB.

\section{Block assessment}

Upon completion of the block, a blinded observer not aware of the injectate volume, assessed the onset of sensory and motor block in the innervation area of each nerve (median, ulnar, radial, and musculocutaneous nerve) every 2.5 mins, until surgical anaesthesia was achieved or 30 mins have elapsed. Sensory function was scored as being present or absent and motor function was graded using the modified Bromage scale (Table I) [4]. Surgical anaesthesia was defined as a motor score $\leq 2$, with absent sensation to cold (tested with ethyl chloride BP, Criogesic ${ }^{\circledR}$, Dr Georg Friedrich Henning, Chemische Fabrik Walldorf GmbH, Walldorf, Germany). Each nerve distribution area was individually assessed, and the sensory and motor onset time was measured separately from conclusion of the block (removal of block needle, $\mathrm{T}_{0}$ ) to attainment of absent sensation to cold and a motor score $\leq 2$, respectively. Overall sensory and motor block onset time was taken from $\mathrm{T}_{0}$ to attainment of surgical anaesthesia in all innervation territories. The block was considered a failure if surgical anaesthesia had not been achieved at $30 \mathrm{mins}$ in one or more of the four nerve distribution areas. In case of block failure, an additional rescue block or conversion to general anaesthesia was planned together with separate analysis of data from those patients. All patients received paracetamol $1 \mathrm{~g}$ and diclofenac sodium $75 \mathrm{mg}$ iv intraoperatively. In case of patient discomfort or upon request, sedation with midazolam to a maximum of $3 \mathrm{mg}$ and/or supplemental analgesia with up to 100 ug fentanyl was provided at the discretion of the attending anaesthesiologist.

Table I. Motor and Sensory Testing

\begin{tabular}{lll}
\hline & Motor test & Sensor test \\
\hline Median nerve & Flexion of radial 3 fingers & Thenar eminence \\
Radial nerve & Extension of wrist & Dorsum of hand \\
Ulnar nerve & Abduction of fingers & Hypothenar eminence \\
Musculocutaneous nerve & Elbow flexion & Over base first metacarpal \\
Modified Bromage scale [4]. & & \\
4 & Full strength in relevant muscle. & \\
3 & Reduced strength but ability to move muscle against resistance & \\
2 & Ability to move relevant muscle group against gravity but not against resistance & \\
1 & Flicker of movement in relevant muscle group & \\
0 & No movement in relevant muscle group & \\
\hline
\end{tabular}


Postoperative analgesia was prescribed around the clock in the form of paracetamol $1 \mathrm{~g}$ po 6 hourly and diclofenac $75 \mathrm{mg}$ po 12 hourly. Oxycodone $10 \mathrm{mg}$ orally 4-6 hourly was administered as rescue analgesia. Postoperatively, sensory and motor function of each nerve was assessed every 15 mins. Sensory and motor duration was measured separately for each nerve from $T_{0}$ to return of sensation to cold and motor power to $\geq 3$, respectively. Overall sensory and motor block offset was defined as return of sensation to cold and motor power (score $\geq 3$ ) respectively, in any one nerve distribution area.

The primary outcome was overall surgical anaesthesia onset time, which was defined as the time elapsed from conclusion of block $\left(\mathrm{T}_{0}\right)$ until attainment of surgical anaesthesia in all nerves distribution areas. Secondary outcome measures included overall duration of sensory and motor block, as well as sensory and motor onset times and durations of individual blocks. Overall duration of surgical anaesthesia was defined as time elapsed from $\mathrm{T}_{0}$ to return of sensation and motor power (score $\geq 3$ ) respectively in any one nerve distribution area.

Block performance parameters were recorded such as imaging time (defined as time elapsed from placement of US probe on the patient to acquisition of a satisfactory image of the axillary artery and surrounding nerves) and needling time (defined as the time interval between insertion and removal of block needle). Thus, performance time was defined as the sum of imaging and needling times. The number of needle passes were recorded. The initial needle pass was considered as the first pass and any subsequent needle advancement preceded by retraction of $1 \mathrm{~cm}$ counted as an additional pass. Incidences of vascular puncture and paraesthesia were also noted.

\section{Sample size and statistical analysis}

In the absence of data from previous studies using $20 \mathrm{ml}$ of lidocaine $2 \%$ with epinephrine for ultrasound guided axillary brachial plexus block, sample size was calculated based on our pilot study of 10 patients. We found a mean \pm SD onset time of $11.25 \pm 2.3 \mathrm{~min}$. The minimum sample size required to have an $80 \%$ probability of detecting a $30 \%$ decrease in onset time (level of significance 0.05 ) was 7 patients per group. We recruited 10 patients per group to account for potential dropouts.

Statistical analysis was performed using SPSS version 24 (IBM, Armonk, New York). The Shapiro-Wilk test was used for normality testing. Continuous, normally distributed data are presented as mean (SD), and nonnormally distributed data as median (interquartile range [IQR]). Comparison between groups were analysed using the unpaired Student's t test for normally distributed data and the Mann-Whitney $U$ test for nonparametric data. Categorical variables were compared between groups using Pearson's or Fischer's exact test. All tests were two-tailed, and $\mathrm{P}<0.05$ was considered statistically significant.

\section{Results}

Twenty patients (10 in each group) were recruited to the study from September 2014 to August 2015. All patients completed the study (fig 1) and none of the patients required rescue block, conversion to general anaesthesia or intraoperative opioid analgesia. There were no adverse events noted in either group. The patient demographic characteristics were similar between the groups (Table II). Table III details onset times. The median [IQR] overall onset time of surgical anaesthesia was shorter in Group 1\% compared to Group 2\%. The overall onset time of sensory but not motor block was also shorter in Group 1\%. Onset times of individual nerves were similar in the two groups, with the exception of median sensory onset time which was shorter in Group 1\%. Table IV depicts block durations. The mean (SD) overall duration of surgical anaesthesia was shorter in Group 1\% compared to Group 2\%, reflective of overall motor block duration. Individual sensory and motor block durations were similar, with median motor block duration shorter in Group $1 \%$. Figure 2 shows the primary outcome measure, overall onset of surgical anaesthesia.

Table II. Patient characteristics

\begin{tabular}{llll}
\hline & Group 2\% (n=10) & Group 1\% (n=10) & p value \\
\hline Age, y & $46.8 \pm 18.2$ & $48 \pm 15.7$ & 0.88 \\
Sex, M/F, n & $7 / 3$ & $8 / 2$ & 0.60 \\
BMI, Kg/m & $25.3 \pm 3.2$ & $24.5 \pm 3.6$ & 0.62 \\
ASA grade (I/II/III), n & $7 / 3 / 0$ & $4 / 6 / 0$ & 0.18 \\
Duration of surgery, min & $62 \pm 10.8$ & $58.5 \pm 14.9$ & 0.56 \\
Site of surgery (wrist/hand), n & $5 / 5$ & $6 / 4$ & \\
\hline
\end{tabular}

Continuous variables are presented as means $\pm \mathrm{SD}$, categorical variables as counts 
Group 1\% had a shorter needling time, performance time and fewer needle passes when compared to Group $2 \%$. No difference was found between the groups with respect to imaging time, incidence of vascular puncture or paraesthesia (Table V).

\section{Discussion}

In this single centre randomised controlled trial, we observed that when using $400 \mathrm{mg}$ of lidocaine with epinephrine, increasing the volume of injectate by dilution

Table III. Sensory and Motor Block Onset Times

\begin{tabular}{|c|c|c|c|}
\hline & $20 \mathrm{ml}$ Lidocaine $2 \% \quad(n=10)$ & $40 \mathrm{~mL}$ Lidocaine $1 \% \quad(n=10)$ & p value \\
\hline Overall sensory onset & $8.75[5-10]$ & $5[5-7.5]$ & 0.046 \\
\hline Overall motor onset & $6.25[5-7.5]$ & $5[2.5-7.5]$ & 0.41 \\
\hline Overall onset of surgical anaesthesia & $8.75[7.5-10]$ & $6.25[5-7.5]$ & 0.03 \\
\hline $\begin{array}{l}\text { Radial } \\
\text { Sensory } \\
\text { Motor }\end{array}$ & $\begin{array}{l}5[5-10] \\
5[2.5-7.5]\end{array}$ & $\begin{array}{l}5[2.5-7.5] \\
3.75[2.5-5]\end{array}$ & $\begin{array}{l}0.12 \\
0.55\end{array}$ \\
\hline $\begin{array}{l}\text { Ulnar } \\
\text { Sensory } \\
\text { Motor }\end{array}$ & $\begin{array}{l}6.25[5-7.5] \\
5[2.5-7.5]\end{array}$ & $\begin{array}{l}5[2.5-5] \\
5[2.5-7.5]\end{array}$ & $\begin{array}{l}0.12 \\
0.87\end{array}$ \\
\hline $\begin{array}{l}\text { Median } \\
\text { Sensory } \\
\text { Motor }\end{array}$ & $\begin{array}{l}6.25[5-10] \\
5[2.5-7.5]\end{array}$ & $\begin{array}{l}5[2.5-5] \\
5[2.5-5]\end{array}$ & $\begin{array}{l}\mathbf{0 . 0 3} \\
0.93\end{array}$ \\
\hline $\begin{array}{l}\text { Musculocutaneous } \\
\text { Sensory } \\
\text { Motor }\end{array}$ & $\begin{array}{l}5[5-10] \\
3.75[2.5-5]\end{array}$ & $\begin{array}{l}3.75[2.5-5] \\
5[2.5-5]\end{array}$ & $\begin{array}{l}0.10 \\
0.87\end{array}$ \\
\hline
\end{tabular}

Values are Median [IQR] expressed in min

Table IV. Sensory and Motor Block Duration

\begin{tabular}{llll}
\hline & Group 2\% $(\mathbf{n = 1 0})$ & Group 1\% (n=10) & p value \\
\hline Overall sensory duration & $171.6 \pm 7.1$ & $158.4 \pm 21.7$ & 0.08 \\
Overall motor duration & $165.1 \pm 5.9$ & $150.9 \pm 17.2$ & $\mathbf{0 . 0 2}$ \\
Overall duration of surgical anaesthesia & $165.1 \pm 5.9$ & $150.9 \pm 17.2$ & $\mathbf{0 . 0 2}$ \\
Radial nerve & & & \\
$\quad$ Sensory & $176.1 \pm 3.7$ & $167.40 \pm 20.4$ & 0.20 \\
$\quad$ Motor & $170.1 \pm 7.2$ & $158.4 \pm 18.6$ & 0.08 \\
Ulnar nerve & & & 0.44 \\
$\quad$ Sensory & $174.6 \pm 3.6$ & $168.9 \pm 22.4$ & 0.15 \\
$\quad 168.6 \pm 8.0$ & $158.4 \pm 19.7$ & \\
Motor & & & 0.14 \\
$\quad$ Sedian nerve & $173.1 \pm 5.9$ & $162.9 \pm 19.9$ & $\mathbf{0 . 0 1}$ \\
$\quad$ Motor & $168.1 \pm 7.4$ & $152.4 \pm 15.4$ & 0.10 \\
Musculocutaneous nerve & & & 0.05 \\
$\quad$ Sensory & $173.1 \pm 7.8$ & $161.4 \pm 19.9$ & \\
$\quad$ Motor & $166.6 \pm 7.3$ & $152.4 \pm 20.4$ & \\
\hline
\end{tabular}

Values are Mean $\pm \mathrm{SD}$, expressed in $\min$

Table V. Block performance data

\begin{tabular}{llll}
\hline & Group 2\% (n=10) & Group 1\% (n=10) & p value \\
\hline Imaging time, min (A) & $2.5 \pm 0.5$ & $2.4 \pm 0.6$ & 0.70 \\
Needling time, min (B) & $8.5 \pm 1.2$ & $6.7 \pm 0.9$ & $\mathbf{0 . 0 0 2}$ \\
Performance time, mins (A+B) & $10.9 \pm 1.3$ & $9.1 \pm 0.5$ & $\mathbf{0 . 0 0 1}$ \\
No. needle passes & $10.5 \pm 2.3$ & $7.2 \pm 1.0$ & $\mathbf{0 . 0 0 1}$ \\
Vascular puncture, n (\%) & $2(20)$ & $1(10)$ & 0.53 \\
Paraesthesia, $\mathrm{n}(\%)$ & $4(40)$ & $2(20)$ & 0.48 \\
\hline
\end{tabular}

Continuous variables are presented as mean $\pm \mathrm{SD}$, categorical variables as count/or percentage. 


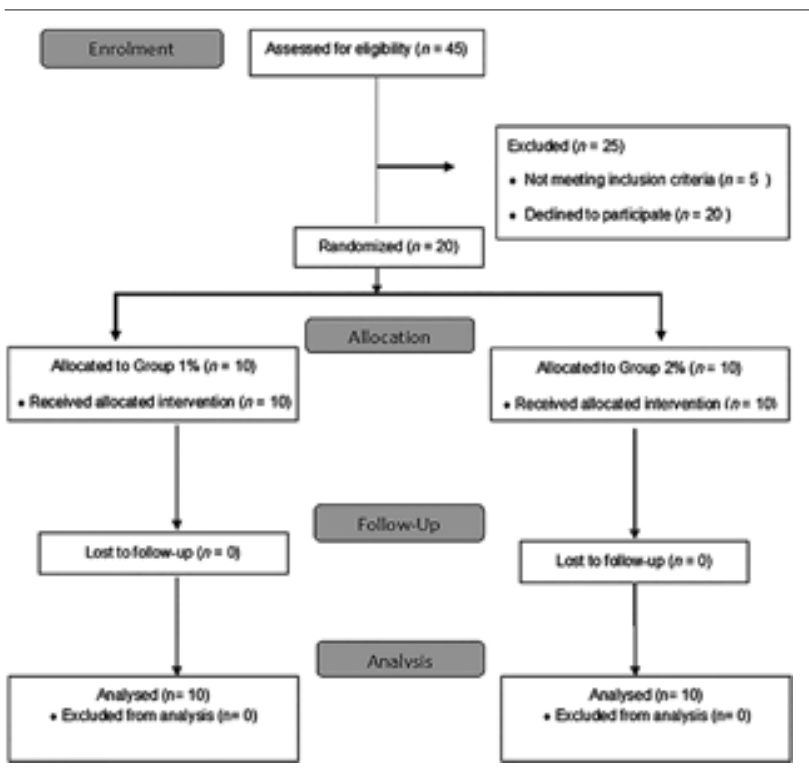

Fig 1. CONSORT patient flow diagram. $\mathrm{n}=$ number

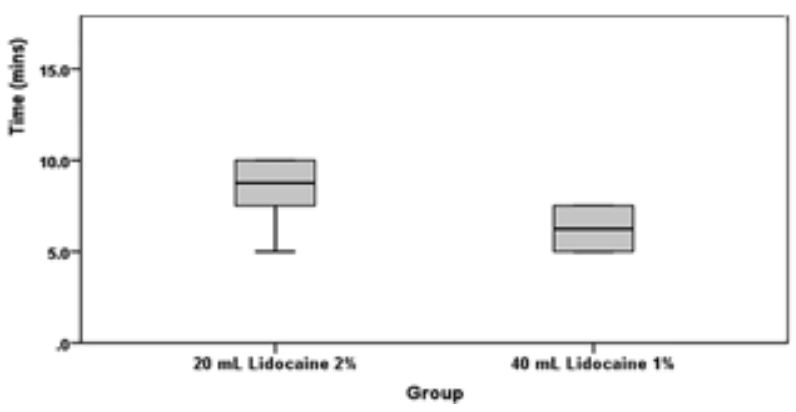

Fig 2. Overall onset of block. The horizontal black line represents the median, the box represents the interquartile range, and the vertical lines show the lowest and highest values. ${ }^{*} \mathrm{p}=0.02$

resulted in shorter overall onset time and subsequent shorter duration of ultrasound guided axillary brachial plexus block. While the shorter onset may be advantageous and desirable in clinical settings with high volume activity and quick turnover, it appears to come at the expense of a shorter duration of block which should be both anticipated and managed appropriately.

In theory, both concentration and volume of the perineural injectate can influence the characteristics of the nerve block. Higher concentrations may shorten the onset time by facilitating the diffusion of local anaesthetic molecules into the nerve [9], while larger volumes may influence the block onset time by promoting injectate spread around neural structures [11]. However, how the volume/concentration ratio at a fixed local anaesthetic dose affects the characteristics of a nerve block, remains unclear. Previous studies yielded inconsistent results with respect to success rate, onset time and duration of the block [8-17]. Several factors such as local anaesthetic volume/concentration ratio, anatomical site of injection and the nerve locating technique used in the study might have contributed to the variable results.

For the Labat approach to the sciatic nerve block using a single injection nerve stimulation technique, Taboada et al observed that $20 \mathrm{~mL}$ of mepivacaine $1.5 \%$ (vs $30 \mathrm{~mL}$ of mepivacaine 1\%) improved the success rate and shortened the onset time of both sensory and motor block [9]. The authors speculated that, because of the size of sciatic nerve and the thickness of epineurium it would require a large concentration gradient to facilitate the diffusion of local anaesthetic molecules. In contrast, Cappelleri et al, using a double injection nerve stimulator technique for sciatic nerve block, found no difference with respect to success rate, onset time and duration of the block between $12 \mathrm{~mL}$ of mepivacaine $2 \%$ and $24 \mathrm{~mL}$ of mepivacaine $1 \%$ [15]. They hypothesized that compared to a single injection technique, the double injection resulted in better distribution of local anaesthetic around each component of the peripheral nerve and with this, the effect of local anaesthetic volume/concentration ratio become secondary to the regional nerve localisation technique.

Similarly, few studies have evaluated the effect of altering the volume and concentration of a fixed local anaesthetic dose for the brachial plexus block. Krenn et al suggested that higher volume of ropivacaine resulted in faster onset of motor block for a single injection axillary block, where loose connective tissue surrounds the brachial plexus [11]. In contrast, studies where the axillary block was performed using the multiple injection nerve stimulator technique [13] and infraclavicular block using ultrasound [14], did not show any difference with respect to block success rate and onset time. In our study, overall onset of surgical anaesthesia was faster using a higher volume when compared to a lower volume (identical dose), and this was mainly reflective of the onset of sensory but not motor component of the block. The difference in the result could be explained by the technique used to locate the target nerves. We performed the ultrasound guided axillary brachial plexus block having identified all four terminal nerves with the precise endpoint consisting of circumferential perineural spread of local anaesthetic, and not using a single or multiple nerve stimulation, or ultrasound guided perivascular approach $[3,18]$.

Interestingly, and perhaps counterintuitively, the injection of the lower volume resulted in a longer block performance time. This is likely due to the requirement for a more precise needle tip positioning and subsequent adjustment in order to achieve circumferential spread around each of the four terminal nerves while having a limited injectate volume at disposal. 
Our study is limited inter alia by the small sample size. Although we found differences between groups in terms of both onset time and duration of block, these results cannot be generalised to other local anaesthetics, techniques and peripheral injection sites due to variation in the anatomical architecture surrounding nerves [19-22]. It has been demonstrated that, using a multiple injection technique for a humeral canal block, higher volume and lower concentration of levobupivacaine improved the sensory block quality and success rate [16]. In contrast, ultrasound guided interscalene block resulted in faster onset of block using lower volume and higher concentration of ropivacaine [17].

In conclusion, when compared to $20 \mathrm{~mL}$ of lidocaine $2 \%$ with epinephrine, $40 \mathrm{~mL}$ of lidocaine $1 \%$ with epinephrine resulted in faster overall onset and shorter duration of surgical anaesthesia following an ultrasound guided axillary brachial plexus block. Further studies are required to determine whether these results can be extrapolated to other local anaesthetics and anatomical injection sites.

Acknowledgement: Assistance with this manuscript: Mr Bahman Honsari provided statistical expertise.

Conflicts of interest: none.

\section{References}

1. Sites BD, Beach ML, Spence BC, et al. Ultrasound guidance improves the success rate of a perivascular axillary plexus block. Acta Anaesthesiol Scand 2006;50:678-684.

2. Chan VW, Perlas A, McCartney CJ, et al. Ultrasound guidance improves success rate of axillary brachial plexus block. Can J Anaesth 2007;54:176-182.

3. Casati A, Danelli G, Baciarello M, et al. A prospective, randomized comparison between ultrasound and nerve stimulation guidance for multiple injection axillary brachial plexus block. Anesthesiology 2007;106:992-996.

4. O'Donnell BD, Iohom G. An estimation of the minimum effective anesthetic volume of $2 \%$ lidocaine in ultrasoundguided axillary brachial plexus block. Anesthesiology 2009;111:25-29.

5. Vester-Andersen T, Eriksen C, Christiansen C. Perivascular axillary block III: blockade following $40 \mathrm{ml}$ of $0.5 \%, 1 \%$ or $1.5 \%$ mepivacaine with adrenaline. Acta Anaesthesiol Scand 1984;28:95-98.

6. Serradell A, Herrero R, Villanueva JA, et al. Comparison of three different volumes of mepivacaine in axillary plexus block using multiple nerve stimulation. Br J Anaesth 2003;91:519-524.

7. Casati A, Fanelli G, Aldegheri G, et al. Interscalene brachial plexus anaesthesia with $0.5 \%, 0.75 \%$ or $1 \%$ ropivacaine: a double-blind comparison with $2 \%$ mepivacaine. Br J Anaesth 1999;83:872-875.

8. Smith BE, Siggins D. Low volume, high concentration block of the sciatic nerve. Anaesthesia 1988;43:8-11.
9. Taboada Muñiz M, Rodríguez J, Bermúdez M, et al. Low volume and high concentration of local anesthetic is more efficacious than high volume and low concentration in Labat's sciatic nerve block: a prospective, randomized comparison. Anesth Analg 2008;107:2085-2088.

10. Vester-Andersen T, Christiansen C, Sørensen M, et al. Perivascular axillary block II: influence of injected volume of local anaesthetic on neural blockade. Acta Anaesthesiol Scand 1983;27:95-98.

11. Krenn H, Deusch E, Balogh B, et al. Increasing the injection volume by dilution improves the onset of motor blockade, but not sensory blockade of ropivacaine for brachial plexus block. Eur J Anaesthesiol 2003;20:21-25.

12. Brenner D, Iohom G, Mahon P, Shorten G. Efficacy of axillary versus infraclavicular brachial plexus block in preventing tourniquet pain. Eur J Anaesthesiol 2019;36:48-54.

13. Bertini L, Palmisani S, Mancini S, et al. Does local anesthetic dilution influence the clinical effectiveness of multiple-injection axillary brachial plexus block?: a prospective, double-blind, randomized clinical trial in patients undergoing upper limb surgery. Reg Anesth Pain Med 2009;34:408-413.

14. González AP, Bernucci F, Techasuk W, et al. A randomized comparison between 3 combinations of volume and concentration of lidocaine for ultrasound-guided infraclavicular block. Reg Anesth Pain Med 2013;38:206-211.

15. Cappelleri G, Ambrosoli AL, Turconi S, et al. Effect of local anesthetic dilution on the onset time and duration of doubleinjection sciatic nerve block: a prospective, randomized, blinded evaluation. Anesth Analg 2014;119:489-493.

16. Nuñez Aguado D, López Alvarez S, Salamanca Montaña $\mathrm{ME}$, et al. [Brachial plexus block with levobupivacaine at the humeral canal: comparison of a small volume at high concentration with a large volume at low concentration]. Rev Esp Anestesiol Reanim 2005;52:529-535.

17. Zhai W, Wang X, Rong Y, Li M, Wang H. Effects of a fixed low-dose ropivacaine with different volume and concentrations on interscalene brachial plexus block: a randomized controlled trial. BMC Anesthesiol 2016;16:80.

18. Bernucci F, Gonzalez AP, Finlayson RJ, Tran DQ. A prospective, randomized comparison between perivascular and perineural ultrasound-guided axillary brachial plexus block. Reg Anesth Pain Med 2012;37:473-477.

19. Thompson GE, Rorie DK. Functional anatomy of the brachial plexus sheaths. Anesthesiology 1983;59:117-122.

20. Areeruk P, Karmakar MK, Reina MA, Mok LYH, Sivakumar RK, Sala-Blanch X. High-definition ultrasound imaging defines the paraneural sheath and fascial compartments surrounding the cords of the brachial plexus at the costoclavicular space and lateral infraclavicular fossa. Reg Anesth Pain Med 2021, DOI:10.1136/rapm-2020-102304.

21. Cornish PB, Leaper CJ, Hahn JL. The "axillary tunnel": an anatomic reappraisal of the limits and dynamics of spread during brachial plexus blockade. Anesth Analg 2007; 104:1288-1291.

22. Cornish PB, Leaper C. The sheath of the brachial plexus: fact or fiction? Anesthesiology 2006;105:563-565. 